\title{
Another nail in the coffin of the cognitive paradigm of dementia ${ }^{\dagger}$
}

Alistair Burns

\section{Summary}

An emerging appreciation by practitioners and researchers, long held by patients and carers, is that neuropsychiatric features of dementia and the challenges they present are as important as those of cognitive losses.

\section{Declaration of interest}

A.B. has received research funding, honoraria and expenses for consultancy work from companies involved in the manufacturing and marketing of drugs for dementia: Baxter, Eisai, Janssen, Lundbeck, Novartis, Pfizer and Shire.
Alistair Burns (pictured) is Professor of Old Age Psychiatry at the University of Manchester and an Honorary Consultant Psychiatrist in the Manchester Mental Health and Social Care Trust.

When Alois Alzheimer described his first patient in $1906^{1}$ she had prominent psychiatric symptoms (delusions that Alzheimer meant her harm) and behavioural problems (screaming). It was the combination of these symptoms and organic brain changes (senile plaques and neurofibrillary tangles identified histologically) in a younger person (she was aged 51 when the disease started and died 4.5 years later) which set the case apart and earned the eponym Alzheimer's disease. Despite these prominent features, the cognitive paradigm of Alzheimer's disease was established with memory and language disorders being regarded as the primary symptoms with psychological and behavioural problems being at the very least secondary, if not merely epiphenomena. It was only in the 1980s and 1990s that the importance of the symptoms became apparent, prompted by recognition of their importance to carers, the development of treatments to control positive symptoms and to curb behaviours, and the ability to make valid measurements of their presence and impact.

\section{Terminology}

Various terms have been invoked to describe essentially the same thing. 'Non-cognitive features' simply distinguished them from the cognitive symptoms of amnesia, aphasia and apraxia but presented a negative connotation, underlining their secondary role. The phrase 'behavioural symptoms' (somewhat of a non sequitur) captured the mood for a while (and still persists), but the more inclusive 'behavioural and psychological symptoms of dementia' has a catchiness about it that neatly separates behaviours and symptoms and emphasises their psychological nature (a psychological reaction to illness and perhaps a way of communicating distress rather than a medical symptom which needs to be suppressed). ${ }^{2}$ The term 'neuropsychiatric features' has been adopted more recently, emphasising the relationship with neurological damage and building on the, now standard, instrument used for their assessment. ${ }^{3}$

\section{Phenomenology and assessment}

Descriptions of the phenomenology of dementia have been etched with reference to general psychiatric psychopathology and so,

†See pp. 212-219, this issue. unsurprisingly, the two have much in common. Until fairly recently, people with dementia did not present until later in their illness and the emphasis on people with more advanced disease led to greater consideration of behavioural disturbances. Later, the full appreciation of the relevance of the range of psychiatric symptoms occurring earlier in the disease emerged, not only positive psychopathology (e.g. delusions and hallucinations), but negative symptoms (e.g. apathy) as well. Personality changes are almost universal and coarsening of affect is often described. What is important is that they have emphasised the key role that emotional changes play as experienced by both the person with dementia and as observed by others.

Although Alzheimer's disease is the paradigm by which other dementias are compared, differences in phenomenology have been documented. Vascular dementia may be associated with increased depression, Lewy body dementia is partly defined by the presence of hallucinations and paranoid ideas, personality change is predominant in frontal lobe dementia, and lack of initiation is a hallmark of the subcortical dementia associated with vascular disease, progressive supranuclear palsy and Parkinson's disease.

Linking psychopathology to biomarkers has been successful in showing correlational rather than causal associations. Associations between general ratings of neuropsychiatric features and brain changes underscore biological mechanisms and have been demonstrated with regional atrophy on computed tomography and magnetic resonance imaging scans, regional hypoperfusion using cerebral blood flow measurements, hypometabolism with positron emission tomography and post-mortem brain changes. Tantalising specific associations include those between symptoms such as wandering behaviour and delusional ideas with regional cerebral metabolic changes, ${ }^{4}$ genetic variations of neurotransmitter receptors with affective symptoms, ${ }^{5}$ and pathological brain changes with a variety of symptoms. ${ }^{6}$

Measurement of neuropsychiatric features has been facilitated by the development of a large number of validated instruments which tend to rely on reports by others rather than direct questioning of people with dementia themselves. ${ }^{7}$ General measures of psychopathology and behaviour are available as well as specific assessments of depression and agitation. The ability to quantify these symptoms has enabled them to become bona fide outcomes in clinical trials.

\section{Epidemiology and aetiology}

There is a general agreement that the symptoms of dementia are very common and probably universal at some point during the course of the illness. Rates of the main symptoms vary with the 
population interviewed, the time period studied (e.g. at any time since diagnosis, or in the past 2 weeks) and the definitions used, but there is general consistency in the estimates of the rates of symptoms. Predictors of later neuropsychiatric features include gender, age, severity of dementia and ApoE4 status. ${ }^{8}$

Several longitudinal studies have now been reported in people with dementia which have emphasised the consistency of a longitudinal course of neuropsychiatric features in dementia. In this issue, a report from the prospective Cognitive Function and Ageing Study (CFAS; Savva et al, 212-219), ${ }^{9}$ the largest study to date of individuals from a representative population both with dementia and who developed dementia over the study period, have shown that mood disturbances and wandering were predicted by baseline scores, that apathy was not associated with sleeping problems, and that agitation and irritability were relatively independent of initial symptoms. Comparing people with and without dementia showed that only sleeping problems were common in the both samples, and four factors were ascertained: psychosis/apathy, irritability/persecution, wandering/ sleep problems and depression/anxiety. Psychosis was associated with declining cognition, and anxiety/ depression was more common in younger people and people with vascular disease. The results have implications for clinical practice: they underscore the omnipresence and unpredictability of apathy; the relative stability of some neuropsychological features and variability of others including the patchy relationship with cognitive impairment which is useful when discussing prognosis in dementia; and the specificity of particular symptoms to dementia which is helpful in the differential diagnosis in the early stages of the disorder. The variability and confounding nature of the associated cognitive deficits should be taken into account when designing prospective clinical trials of neuropsychiatric features; these defecits may also help target groups at risk while undergoing preventive interventions. The findings build on previous studies and their significance is all the more because they are based on a representative group, compared with previous studies that relied on convenient samples of patients attending clinics with all the attendant bias those can bring.

\section{Management}

Traditionally, the suppression of these symptoms with drugs has been the mainstay of treatment and has been successful. However, older people and those with organic brain disease are more susceptible to side-effects and recent studies have called into question the safety of these medicines in people with dementia. In addition to causing increased cognitive decline, antipsychotics have been associated with increased rates of mortality and stroke. ${ }^{10}$ Studies suggest that cerebrovascular side-effects are greater with the atypical antipsychotics, although mortality is the same for both typical and atypical agents. Overprescribing of antipsychotics to control behavioural disturbances in general is inappropriate, particularly as the perception is that this compensates for a shortfall in skilled care staff. Non-pharmacological treatments are potentially safer and usually tried first in practice especially when symptoms are mild. Treatments should be targeted at specific symptoms and time limited. ${ }^{11}$

One of the most consistent findings in caregiver research is that neuropsychiatric features are powerful predictors of carer strain and institutionalisation of people with dementia. ${ }^{12}$ Interventions for carers are effective at reducing burden but the exact nature of that intervention is less crucial in determining success. Research has inevitably concentrated on the later stages of disease where behavioural problems are more apparent; however, emotional changes in the early stages of the disease and negative symptoms such as apathy can be as distressing.

\section{Conclusion}

An emerging appreciation by practitioners and researchers, long held by patients and carers, is that neuropsychiatric features of dementia and the challenges they present are just as important as those of cognitive losses. The description and documentation of the neuropsychiatry of dementia and its subsequent effect on people with dementia and their carers has increased in scope, size and sophistication over the past two decades. What conclusions can be drawn in the light of this evidence?

First (and foremost), there is now a full acceptance that they are an integral part of, and therefore a respectable target for, interventions aimed at improving quality of life in people with dementia and their carers. Second, their natural history is fairly consistent and their occurrence reasonably predictable but less so than the deterioration seen with cognitive function - this leads to the possibility of their (secondary) prevention. The paper by Savva et al in this issue of the Journal supports this contention in a unique prospectively studied population. Third, it shows that in a comparatively short time, an illness phenotype can metamorphose from an idiosyncratic characteristic of little significance into a major marker of the illness, the target of biological and non-biological treatments and a sought-after clinicopathological correlate. What next in dementia, one wonders?

Alistair Burns, Department of Psychiatry, University of Manchester, Manchester M13 9PL, UK. Email: alistair.burns@manchester.ac.uk

First received 15 Aug 2008, final revision 21 Oct 2008, accepted 6 Nov 2008

\section{References}

1 Maurer K, McKeith I, Cummings J, Ames D, Burns A. Has the management of Alzheimer's disease changed over the past 100 years? Lancet 2006; 368: 1619-21.

2 Finkel S, Burns A. Behavioural and psychological symptoms of dementia (BPSD): a clinical and research update. Int Psychogeriatr 2000; 12 (suppl 1): 13-9.

3 Cummings JL, Mega M, Gray K, Rosenberg-Thompson S, Carusi DA, Gornbein J. The Neuropsychiatric Inventory: comprehensive assessment of psychopathology in dementia. Neurology 1994; 44: 2308-14.

4 Mentis MJ, Weinstein EA, Horwitz B, McIntosh AR, Pietrini P, Alexander GE, et al. Abnormal brain glucose metabolism in the delusional misidentification syndromes: a positron emission tomography study in Alzheimer disease. Biol Psychiatry 1995; 38: 438-49.

5 Holmes C, Arranz MJ, Powell JF, Collier DA, Lovestone S. 5-HT receptor polymorphisms and psychopathology in late onset Alzheimer's disease. Hum Mol Genet 1998; 7: 1507-9.

6 Förstl H, Burns A, Levy R, Cairns N. Neuropathological correlates of psychotic phenomena in confirmed Alzheimer's disease. Br J Psychiatry 1994; 165 53-9

7 Burns A, Craig S, Lawlor B. Assessment Scales in Old Age Psychiatry (2nd edn). Martin Dunitz, 2004.

8 Steinberg M, Corcoran C, Tschanz JT, Huber C, Welsh-Bohmer K, Norton MC, et al. Risk factors for neuropsychiatric symptoms in dementia: the Cache County Study. Int J Geriatr Psychiatry 2006; 21: 824-30.

9 Savva GM, Zaccai J, Matthews FE, Davidson JE, McKeith I, Brayne C, et al. Prevalence, correlates and course of behavioural and psychological symptoms of dementia in the population. Br J Psychiatry 2009; 174: 212-9.

10 Douglas IJ, Smeeth L. Exposure to antipsychotics and risk of stroke: self controlled case series study. BMJ 2008; 337: a1227.

11 O'Brien J. Antipsychotics for people with dementia. BMJ 2008; 337: a602.

12 Donaldson C, Burns A. Burden of Alzheimer's disease: helping the patient and caregiver. J Geriatr Psychiatry Neurol 1999; 12: 21-8. 